\title{
SPECIAL $\alpha$-LIMIT POINTS FOR MAPS OF THE INTERVAL
}

\author{
MICHAEL W. HERO
}

(Communicated by Andrew M. Bruckner)

\begin{abstract}
The notion of a special $\alpha$-limit point is defined. For maps of the interval, it is shown that a point is a special $\alpha$-limit point if and only if it is an element of the attracting center.
\end{abstract}

\section{INTRODUCTION}

Our main result is that for continuous self-maps of an interval, the attracting center and the set of special $\alpha$-limit points coincide. Also, combining the work in this paper with that of Xiong in [9], we will obtain a necessary and sufficient condition for the existence of a periodic point with period not a power of two.

Throughout this paper, $f$ will be a continuous self-map of an interval $I$. For every positive integer $n, f^{n}$ will denote the function $f$ composed with itself $n$ times. Using the forward orbit of a point $x$, the $\omega$-limit set $\omega(x)$ of $x$ is defined to be the collection of all limit points of the sequence $\left(f^{n}(x)\right)_{n=1}^{\infty}$. Elements of $\omega(x)$ are called $\omega$-limit points. A point $z$ is said to be recurrent if $z \in \omega(z)$.

In [9] the $\alpha$-limit set $\alpha(x)$ is defined for self-maps of the interval. In this paper we study the special $\alpha$-limit set of a point $x$ and denote it by $s \alpha(x)$. A point $y$ is an element of the set $s \alpha(x)$ provided there exists a sequence of positive integers $(n(i))_{i=1}^{\infty}$ and a sequence of points $\left(y_{i}\right)_{i=0}^{\infty}$ such that

(1) $x=y_{0}$,

(2) $f^{n(i)}\left(y_{i}\right)=y_{i-1}$,

(3) $\lim _{i \rightarrow \infty} y_{i}=y$.

Note that if such a sequence exists then $y$ is an element of $s \alpha\left(y_{i}\right)$ for every $i$.

In order to state our theorem, we must define what is called the attracting center of a dynamical system. For a subset $Y$ of $I$, define $\Lambda(Y)=\bigcup_{x \in Y} \omega(x)$. Let $\Lambda^{1}=\Lambda(I)$. For every $n>1$, inductively define $\Lambda^{n}=\Lambda\left(\Lambda^{n-1}\right)$. The attracting center $\Lambda^{\infty}$ is then the intersection of the sets $\Lambda^{n}$.

Received by the editors November 19, 1990 and, in revised form, April 17, 1991.

1991 Mathematics Subject Classification. Primary 26A18, 58F08; Secondary 54H20, 58F13.

The results in this paper are from the author's Ph.D. thesis, which was completed under the supervision of Professor Richard J. O'Malley at the University of Wisconsin-Milwaukee.

This paper was presented at the South Bend, Indiana, AMS Meeting, March 15, 1991. 
Theorem. Suppose that $f$ is a continuous self-map of an interval I. Then the following are equivalent:

(1) $x \in s \alpha(y)$ for some $y$,

(2) $x \in s \alpha(x)$,

(3) $x \in \Lambda^{\infty}$.

Combining this theorem with the work of Xiong [9], we obtain the following corollary. The period of a periodic point $x$ is the least $n$ such that $f^{n}(x)=x$.

Corollary. The following are equivalent:

(1) Some point $y$ that is not recurrent is a special $\alpha$-limit point.

(2) Some periodic point has period that is not a power of two.

A weaker form of recurrence than that of being an $\omega$-limit point is that of being a nonwandering point. Let $y \in I$. Then $y$ is a nonwandering point if for every open neighborhood $V_{y}$ of $y, V_{y} \cap f^{m}\left(V_{y}\right) \neq \varnothing$ for some $m$.

A pointwise definition of the set of nonwandering points can be obtained by considering the backwards orbit of a point. A point $y$ is an element of $\alpha(x)$ provided that a strictly increasing sequence of positive integers $(n(i))_{i=1}^{\infty}$ and a sequence of points $\left(y_{i}\right)_{i=1}^{\infty}$ can be found such that

(1) $f^{n(i)}\left(y_{i}\right)=x$,

(2) $\lim _{i \rightarrow \infty} y_{i}=y$.

Elements of $\alpha(x)$ are called $\alpha$-limit points. The sets of all $\alpha$-limit points, in general, strictly contains the set of nonwandering points. A pointwise definition of the set $\Omega$ of nonwandering points, however, can be obtained using

Proposition A [6]. If $f$ is a self-map of an interval $I$ then $x \in \Omega$ if and only if $x \in \alpha(x)$.

We now provide an example of a self-map of $[0,4]$ for which $\alpha(1) \neq s \alpha(1)$. A smooth version of this example is used in [7] to show that the point 1 is a nonwandering point but not an $\omega$-limit point. We will see later that if $y$ is a nonwandering point that is not an $\omega$-limit point then $y \in \alpha(y)-s \alpha(y)$. According to the theorem, when $\Lambda^{1} \neq \Lambda^{2}$ an $\omega$-limit point $y$ can be found with $y \in \alpha(y)-s \alpha(y)$.

Example.

$$
f(x)= \begin{cases}x+2 & \text { if } x \in[0,1), \\ 3 x & \text { if } x \in\left[1, \frac{4}{3}\right), \\ -\frac{9}{2} x+10 & \text { if } x \in\left[\frac{4}{3}, 2\right), \\ 2 x-3 & \text { if } x \in\left[2, \frac{7}{2}\right), \\ 4 & \text { if } x \in\left[\frac{7}{2}, 4\right] .\end{cases}
$$

It is clear from the graph of the function $f$ that there is a strictly increasing sequence of points $\left(w_{i}\right)_{i=1}^{\infty}$ with $w_{1}=2, w_{i} \in(2,3)$ and $f\left(w_{i}\right)=w_{i-1}$ for $i>$ 1. For every $i \geq 1$ there is a $z_{i} \in[0,1]$ with $f\left(z_{i}\right)=w_{i}$. Thus $f^{i+1}\left(z_{i}\right)=1$ and $\lim _{i \rightarrow \infty} z_{i}=1$, hence $1 \in \alpha(1)$. The point 1 , however, cannot be a special $\alpha$-limit point since $f^{n}\left(\left[1, \frac{14}{9}\right]\right)=[3,4]$ for every $n>0$ and $f^{-1}([0,1))=\varnothing$. To see this, one needs only to realize that if 1 were a special $\alpha$-limit point, we could find points $y_{1}, y_{2}$, and $y_{3}$ all elements of $\left(0, \frac{14}{9}\right)$ and integers $n(3)$ and $n(2)$ with $f^{n(3)}\left(y_{3}\right)=y_{2}$ and $f^{n(2)}\left(y_{2}\right)=y_{1}$. 


\section{Preliminaries}

A point $y$ is said to be a $\gamma$-limit point of $x$ if $y \in \omega(x) \cap \alpha(x)$. Let $\gamma(x)=\omega(x) \cap \alpha(x)$ and $\Gamma=\bigcup_{x \in I} \gamma(x)$. It follows from Proposition $A$ that the set of recurrent points $R$ is contained in the set $\Gamma$. In his investigation of the set $\Lambda^{\infty}$, Xiong [9] proves the following theorem.

Theorem A. Suppose that $f$ is a continuous self-map of an interval. Then

(1) $\Gamma=\Lambda^{2}=\Lambda^{\infty} \subseteq \bar{P}$, where $P$ is the set of periodic points;

(2) $R=\Gamma$ if and only if the period of every periodic point is a power of 2 , where $R$ is the set of all recurrent points.

A function $f$ displays a horseshoe if there are disjoint closed intervals $J$ and $K$ in the interval and a positive integer $n$ such that $f^{n}(J) \supset J, f^{n}(K) \supset K$, $f^{n}(K) \supset J$, and $f^{n}(J) \supset K$. The content of statement (2) of Theorem A is that $\Gamma$ strictly contains $R$ if and only if $f$ displays a horseshoe. In general, the following set relation holds for self-maps of the interval

$$
P \subseteq R \subseteq \Gamma \subseteq \bar{P} \subseteq \Lambda^{1} \subseteq \Omega \text {. }
$$

In the above example, the set $\Lambda^{1}$ is strictly contained in $\Omega$. For an example of a function where $\bar{P}$ is strictly contained in $\Lambda^{1}$ see [2]. In [3] an example of a function with $\Gamma \neq \bar{P}$ is constructed.

\section{Proof of THE THEOREM}

We will prove several lemmas from which the theorem will follow immediately.

Lemma 1. If $x \in s \alpha(y)$ then $f^{n}(x) \in s \alpha(y)$ for every $n$ and $\omega(x) \subseteq s \alpha(y)$.

Proof. Suppose that $x \in s \alpha(y)$. Then we can find $y_{i}$ with $\lim _{i \rightarrow \infty} y_{i}=x$ and $n(i)>0$ such that $f^{n(i)}\left(y_{i}\right)=y_{i-1}$ and $f^{n(1)}\left(y_{1}\right)=y$.

Consider the sequence

$$
\begin{aligned}
& \left(y, f^{n(1)-1}\left(y_{1}\right), \ldots, y_{1}, f^{n(2)-1}\left(y_{2}\right), \ldots, y_{2}, f^{n(3)-1}\left(y_{3}\right), \ldots\right) \\
& \quad \equiv\left(y, z_{1}, z_{2}, z_{3}, \ldots\right) .
\end{aligned}
$$

Note that if $m<\sum_{i=1}^{j} n(i)$ then $f^{m}\left(y_{j}\right)$ is a term of the sequence $\left(z_{i}\right)_{i=1}^{\infty}$. One can see that any limit point of the sequence $\left(z_{i}\right)_{i=1}^{\infty}$ is an element of $s \alpha(y)$.

Let $z \in\left\{f(x), f^{2}(x), \ldots\right\} \cup \omega(x)$ and let $\varepsilon>0$ be given. Then we can find an $m>0$ with $f^{m}(x) \in(z-\varepsilon, z+\varepsilon)$. The function $f^{m}$ is continuous and $\lim _{i \rightarrow \infty} y_{i}=x$, thus for some $N>0$ if $j>N$ then $f^{m}\left(y_{j}\right) \in(z-\varepsilon, z+\varepsilon)$. If we take $j>\max (N, m)$ then $m<j \leq \sum_{i=1}^{j} n(i)$. Thus $f^{m}\left(y_{j}\right) \in(z-\varepsilon, z+\varepsilon)$ and is a term of the sequence $\left(z_{i}\right)_{i=1}^{\infty}$. It follows that $z$ is a limit point of the sequence $\left(z_{i}\right)_{i=1}^{\infty}$.

To state Lemma 2, we develop some notation. We will use the symbols $\alpha_{R}(x)$ (resp., $\alpha_{L}(x)$ ) to denote the set of all points $y$ such that there exists a sequences $(n(i))_{i=1}^{\infty}$ and $\left(x_{i}\right)_{i=1}^{\infty}$ with $\lim _{i \rightarrow \infty} n(i)=\infty, \lim _{i \rightarrow \infty} x_{i}=y, f^{n(i)}\left(x_{i}\right)=x$, and $y<x_{i}$ (resp., $x_{i}<y$ ) for every $i>0$.

Lemma 2. (1) If for some $\varepsilon>0, x \in \alpha_{L}(y)$ for every $y \in(x-\varepsilon, x)$, then for every $y \in(x-\varepsilon, x), x \in s \alpha(y)$. 
(2) If for some $\varepsilon>0, x \in \alpha_{R}(y)$ for every $y \in(x, x+\varepsilon)$, then for every $y \in(x, x+\varepsilon), x \in s \alpha(y)$.

Proof. We will prove (1). Let $y \in(x-\varepsilon, x)$. Since $x \in \alpha_{L}(y)$, we can find $y_{1} \in$ $(x-\varepsilon / 2, x)$ and $n(1)>0$, with $f^{n(1)}\left(y_{1}\right)=y$. By hypothesis, $x \in \alpha_{L}\left(y_{1}\right)$; thus we can find $y_{2} \in\left(x-\varepsilon / 2^{2}, x\right)$ and $n(2)>0$, with $f^{n(2)}\left(y_{2}\right)=y_{1}$. Continuing in this way, we obtain a sequence $\left(y_{i}\right)_{i=1}^{\infty}$ and $n(i)>0$, with $y_{i} \in\left(x-\varepsilon / 2^{i}, x\right)$ and $f^{n(i)}\left(y_{i}\right)=y_{i-1}$.

Before proceeding any further, we need the definitions of almost periodic points and minimal sets. If given an open set $V_{y}$ containing $y$, one can find an integer $n>0$ such that for any integer $q>0$ there is an integer $r, q \leq r \leq q+n$ with $f^{r}(y) \in V_{y}$, then $y$ is said to be almost periodic. If for every $x \in \omega(y)$ we have that $\omega(x)=\omega(y)$, then $\omega(y)$ is said to be a minimal set. A well-known fact is that for compact metric spaces a point $y$ is almost periodic if and only if $y \in \omega(y)$ and $\omega(y)$ is a minimal set.

Lemma 3. If $x$ is almost periodic then $x \in s \alpha(x)$.

Proof. Since $x$ is almost periodic, $x \in \omega(x)$ and $\omega(x)$ is a minimal set. Since $f(\omega(x))=\omega(x)$, we can find a sequence of points $\left(z_{i}\right)_{i=1}^{\infty}$ with $z_{i} \in \omega(x)$, $f\left(z_{i}\right)=z_{i-1}$, and $f\left(z_{1}\right)=x$. Let $y$ be a limit point of this sequence. Then $y \in \operatorname{s\alpha }(x)$ and, since $\omega(x)$ is a closed set, $y \in \omega(x)$. Since in this case $\omega(x)$ is a minimal set, $x \in \omega(y)$. It follows from Lemma 1 that $x \in s \alpha(x)$.

Let $Y$ be a subset of $I$. We will denote the closure of $Y$ by $\bar{Y}$. A point $z$ is called a right-side (resp., left-side) accumulation point of $Y$ if for every $\varepsilon>0,(z, z+\varepsilon) \cap Y \neq \varnothing$ (resp., $(z-\varepsilon, z) \cap Y \neq \varnothing)$. The right-side closure $\bar{Y}_{R}$ (resp., left-side closure $\bar{Y}_{L}$ ) is the union of $Y$ and the set of right-side (resp., left-side) accumulation points of $Y$.

In Lemma 4 bilateral limit points of periodic points are considered. We will see that such points are special $\alpha$-limit points. If in addition the point $x$ in question is not almost periodic, then we show that $x$ is the end point of interval $J$ with $x \in s \alpha(y)$ for every $y \in J$. In either case we will see that $x \in s \alpha(x)$.

Lemma 4. If $x \in \bar{P}_{L} \cap \bar{P}_{R}$ then $x \in s \alpha(x)$. If in addition $x$ is not almost periodic, then for some $\varepsilon>0$, either $x \in s \alpha(y)$ for every $y \in(x-\varepsilon, x]$, or $x \in s \alpha(y)$ for every $y \in[x, x+\varepsilon)$.

Proof. According to Lemma 3, we may assume that $x$ is not almost periodic. If for every $\varepsilon>0$ we could find an $n>0$ such that $f^{n k}(x) \in(x-\varepsilon, x+\varepsilon)$ for every $k$, then $x$ would be almost periodic. Thus we can find an $\varepsilon>0$ such that if $n>0$ is fixed then for some $k>0, f^{n k}(x) \notin(x-\varepsilon, x+\varepsilon)$.

Fix such an $\varepsilon>0$. By our hypothesis, $\lim _{i \rightarrow \infty} p_{i}=x, \lim _{i \rightarrow \infty} q_{i}=x$ with $p_{i}<x$ and $x<q_{i}$ for two sequences of periodic points $\left(p_{i}\right)_{i=1}^{\infty}$ and $\left(q_{i}\right)_{i=1}^{\infty}$. Furthermore, we may assume that $p_{i} \in(x-\varepsilon, x)$ and $q_{i} \in(x, x+\varepsilon)$. Let $n(i)$ be the period of $p_{i}$ and $m(i)$ the period of $q_{i}$. Let $l(i)=n(i) m(i)$. It follows from our choice of $\varepsilon$ that we can find $k(i)>0$ with $f^{l(i) k(i)}(x) \notin(x-\varepsilon, x+\varepsilon)$.

Suppose by choosing a subsequence, if needed, that $f^{l(i) k(i)}(x)<x-\varepsilon$ for every $i$. Since the period of $p_{i}$ is $n(i), f^{l(i) k(i)}\left(p_{i}\right)=p_{i}$; thus

$$
(*) \quad\left(x-\varepsilon, p_{i}\right] \subseteq f^{l(i) k(i)}\left(\left[p_{i}, x\right)\right) \text {. }
$$

Let $y \in(x-\varepsilon, x)$. We will now show that $x \in s \alpha(y)$. Since $\lim _{i \rightarrow \infty} p_{i}=x$, we can find a positive integer $i(1)$ such that $x-\varepsilon<y<p_{i(1)}<x$. It follows 
from $(*)$ that there exists $z_{i(1)} \in\left(p_{i(1)}, x\right)$ with $f^{l(i(1)) k(i(1))}\left(z_{i(1)}\right)=y$. Since $z_{i(1)} \in\left(p_{i(1)}, x\right)$, we can find a positive integer $i(2)$ with

$$
x-\varepsilon<y<p_{i(1)}<z_{i(1)}<p_{i(2)}<x .
$$

It follows from $(*)$ that there exists $z_{i(2)} \in\left(p_{i(2)}, x\right)$ with $f^{l(i(2)) k(i(2))}\left(z_{i(2)}\right)=$ $z_{i(1)}$. Continuing in this way, it is possible to find $i(j), p_{i(j)}$, and $z_{i(j)}$ with

$$
x-\varepsilon<y<p_{i(1)}<z_{i(1)}<p_{i(2)}<\cdots<p_{i(j)}<z_{i(j)} \cdots<x
$$

such that

$$
f^{l(i(j)) k(i(j))}\left(z_{i(j)}\right)=z_{i(j-1)} .
$$

Since $\lim _{i \rightarrow \infty} p_{i}=x$, we have that $\lim _{j \rightarrow \infty} z_{i(j)}=x$. Thus $x \in s \alpha(y)$.

To finish the proof of the lemma it suffices to show that we can find a point $z \in(x-\varepsilon, x)$ and an $n>0$ with $f^{n}(z)=x$. The argument that we will give here is taken from the proof of Proposition $C$ in [9]. Let $g=f^{n(1)}$ and $L=\left[p_{1}, x\right]$. Then since $g\left(p_{1}\right)=p_{1}, K=L \cup g(L) \cup g^{2}(L) \cup \cdots$ is an interval. Let $l(j)$ denote the period of $p_{j}$ with respect to $g$. For $k=1,2$, or 3 suppose that a subsequence of $g^{l(1)-k}\left(p_{1}\right) g^{l(2)-k}\left(p_{2}\right) \cdots g^{l(j)-k}\left(p_{j}\right) \cdots$ converges to $u_{k} \in \bar{K}$. It is clear that $g^{k}\left(u_{k}\right)=x$. If $u_{k^{\prime}}=u_{k^{\prime \prime}}$ for some $k^{\prime}<k^{\prime \prime}$, then

$$
g^{k^{\prime \prime}-k^{\prime}}(x)=g^{k^{\prime \prime}-k^{\prime}}\left(g^{k^{\prime}}\left(u_{k^{\prime}}\right)\right)=g^{k^{\prime \prime}}\left(u_{k^{\prime}}\right)=g^{k^{\prime \prime}}\left(u_{k^{\prime \prime}}\right)=x .
$$

However, $x$ is by assumption not periodic, thus we must have that $u_{1}, u_{2}$, and $u_{3}$ are distinct points in $\bar{K}$. Since $K$ is an interval, $u_{k} \in K$ for some $k$. Hence for some point $z \in L$ and $n \geq 0, g^{n}(z)=u_{k}$. Thus $g^{n+k}(z)=x$.

Note that the last argument in the proof of Lemma 4 shows that for points $x \in \bar{P}_{L}-P$ (resp., $\bar{P}_{R}-P$ ), we have $x \in \alpha_{L}(x)$ (resp., $\left.\alpha_{R}(x)\right)$. We will use the symbols $\omega_{R}(x)$ (resp., $\left.\omega_{L}(x)\right)$ to denote the set of right-side (resp., left-side) accumulation points of the set $\left\{f^{n}(x)\right\}_{n=1}^{\infty}$.

Lemma 5. If $x \in R$ then $x \in s \alpha(x)$.

Proof. Let $x \in R$. If $\omega(x)$ is a minimal set, then according Lemma $3, x \in$ $s \alpha(x)$.

We assume that $\omega(x)$ is not a minimal set. Applying Lemma 3 of [1] to the compact set $\omega(x)$, we have that the set $A=\{y \mid \omega(y)=\omega(x)\} \cap \omega(x)$ is an uncountable set. Let $\Delta$ denote the symmetric difference of sets. Since $\bar{P}_{L} \Delta \bar{P}_{R}$ and $\omega_{L}(x) \Delta \omega_{R}(x)$ are both countable, $\bar{P}_{L} \cap \bar{P}_{R}$ (resp., $\omega_{L}(x) \cap \omega_{R}(x)$ ) is the complement of a countable set in $\bar{P}$ (resp., $\omega(x)$ ); since $\omega(x) \subseteq \bar{P}$, it follows that

$$
\omega_{L}(x) \cap \omega_{R}(x) \cap \bar{P}_{L} \cap \bar{P}_{R}
$$

is the compliment of a countable set in $\omega(x)$. But then $A$, being an uncountable subset of $\omega(x)$, must intersect the set $\omega_{L}(x) \cap \omega_{R}(x) \cap \bar{P}_{L} \cap \bar{P}_{R}$. Let $y$ be a point from $A$ in this intersection.

Since $\omega(x)=\omega(y)$ and $\omega(x)$ is not a minimal set, it must be that $y$ is not almost periodic. Without loss of generality, by Lemma 4 we may choose an $\varepsilon>0$ such that if $z \in(y-\varepsilon, y]$ then $y \in s \alpha(z)$. Now $y \in \omega_{L}(x)$; hence for some positive integer $n, f^{n}(x) \in(y-\varepsilon, y)$. Since the function $f^{n}$ is continuous, for some $\delta>0$

$$
f^{n}((x-\delta, x+\delta)) \subseteq(y-\varepsilon, y)
$$


Now $R \subseteq \Omega$, so by Proposition A, $x \in \alpha(x)$. Thus for some $z \in(x-\delta, x+\delta)$ and $m>n, f^{m}(z)=x$. By $(* *), f^{n}(z) \in(y-\varepsilon, y)$, hence $y \in s \alpha\left(f^{n}(z)\right)$ and $f^{m-n}\left(f^{n}(z)\right)=x$. It follows that $y \in s \alpha(x)$. Since $x \in \omega(y)$, by Lemma $1, x \in s \alpha(x)$.

We consolidate our proof that (1) implies (2) implies (3) in our theorem into the proof of Proposition 1. Before we prove Proposition 1, two well-known results must be stated. The first, Lemma $B$ is a description of the dynamical behavior that can occur on subintervals of $I$ containing no periodic points. The second, Proposition $\mathrm{C}$, is a description of a situation under which one can conclude that a point is an element of $\omega_{R}(x)$ (resp., $\omega_{L}(x)$ ) for some $x$. For a proof of Lemma B see [5] and for Proposition C see [4]. Proposition C is due to Sharkovskiǐ in [8].

Lemma B. Let $J$ be a subinterval of I that does not contain any periodic points. If $x$ and $y$ are elements of $J$ and iffor some $n$ and $m$ both $f^{n}(x)$ and $f^{m}(y)$ are elements of $J$, then either $f^{n}(x)<x$ and $f^{m}(y)<y$, or $f^{n}(x)>x$ and $f^{m}(y)>y$.

Proposition C. If for every $\varepsilon>0$, an $n>0$ can be found with

$$
(c, c+\varepsilon) \cap f^{n}((c, c+\varepsilon)) \neq \varnothing
$$

(resp., $\left.(c-\varepsilon, c) \cap f^{n}((c-\varepsilon, c)) \neq \varnothing\right)$, then $c \in \omega_{R}(y) \cup P\left(\right.$ resp., $\left.c \in \omega_{L}(y) \cup P\right)$ for some $y \in I$.

Proposition 1. If $x \in s \alpha(y)$ for some $y$ then $x \in s \alpha(x)$ and $x \in \Gamma$.

Proof. Let $x \in s \alpha(y)$. Since $R \subseteq \Gamma$, by Lemma 5 we may assume that $x$ is not recurrent.

Fix an $\varepsilon>0$ such that $f^{n}(x) \notin(x-\varepsilon, x+\varepsilon)$ for every $n>0$. As $x \in s \alpha(y)$ we can find a sequence $\left(y_{i}\right)_{i=1}^{\infty}$ and $n(i)>0$ with $\lim _{i \rightarrow \infty} y_{i}=x$ and $f^{n(i)}\left(y_{i}\right)=y_{i-1}$. By choosing a subsequence of the sequence $\left(y_{i}\right)_{i=1}^{\infty}$, we may assume that $y_{i} \in(x-\varepsilon, x)$ for every $i>0$.

Claim 1. The point $x$ is an element of $\bar{P}_{L}-P$ and hence, as noted above, an element of $\alpha_{L}(x)$.

Proof of Claim 1. Let $\delta>0$ be given. It is possible to find a $j>0$ with $y_{j}$ and $y_{j-1}$ both elements of $(x-\delta, x)$ and hence, $(x-\delta, x) \cap f^{n(j)}((x-\delta, x)) \neq \varnothing$. Since $x$ is not periodic by Proposition C, $x \in \omega_{L}(y)$ for some $y$. Now if, on the contrary, $x \notin \bar{P}_{L}$ then we can find an $\bar{\varepsilon}>0$ such that $(x-\bar{\varepsilon}, x) \cap P=\varnothing$. Since $x \in \omega_{L}(y)$, we can assume that $y \in(x-\varepsilon, x)$. Thus for some $n>0$, $x-\varepsilon<y<f^{n}(y)<x$. Since $\lim _{i \rightarrow \infty} y_{i}=x$, we can find an integer $j$ with $x-\bar{\varepsilon}<y_{j-1}<y_{j}<x$. This is, however, a contradiction to Lemma B as $f^{n(j)}\left(y_{j}\right)=y_{j-1}$. Thus $x \in \bar{P}_{L}-P$ and hence $x \in \alpha_{L}(x)$.

Claim 2. For some $\delta>0, x \in s \alpha(y)$ for every $y \in(x-\delta, x]$.

Proof of Claim 2. To prove this claim we first define the integers $M(k, j)$ for every $k>0$ and $j>0$, according to the equation $M(k, j)=\sum_{i=1}^{j} n(k+i)$. Since $f^{n(i)}\left(y_{i}\right)=y_{i-1}$, we have that $f^{M(k, j)}\left(y_{k+j}\right)=y_{k}$. We consider two cases. 
Case 1. For some $k>0, x+\varepsilon<f^{M(k, j)}(x)$ for an infinite number of $j$. Fix such a $k>0$. Let $(h(i))_{i=1}^{\infty}$ be an increasing subsequence of positive integers such that $x<f^{M(k, h(i))}(x)$ for every $i>0$. In this case we will show that for every $y \in\left(y_{k}, x\right], x \in s \alpha(y)$. Since $x \in \alpha_{L}(x)$, by Lemma 2 it suffices to show that $x \in \alpha_{L}(y)$ for every $y \in\left(y_{k}, x\right)$. So let $y \in\left(y_{k}, x\right)$. For every $i>0$,

$$
\left[y_{k}, f^{M(k, h(i))}(x)\right] \subseteq f^{M(k, h(i))}\left(\left[y_{k+h(i)}, x\right]\right) .
$$

Hence for every $i>0$ we can find a point $z_{i} \in\left[y_{k+h(i)}, x\right]$ with $f^{M(k, h(i))}\left(z_{i}\right)$ $=y$. Since $\lim _{i \rightarrow \infty} y_{k+h(i)}=x$, it follows that $x \in \alpha_{L}(y)$.

Case 2. For every $k>0$, we can find a positive integer $M(k)$ so that if $j>$ $M(k)$ then $f^{M(k, j)}(x)<x-\varepsilon$. In this case we will prove that for $y \in(x-\varepsilon, x)$, $x \in \alpha_{L}(y)$. Let $y \in(x-\varepsilon, x)$. We can find a $k>0$ with $y<y_{k}$. By our assumption for $j>M(k)$, we have

$$
f^{M(k, j)}(x)<x-\varepsilon<y<y_{k} .
$$

It follows that for every $j>M(k)$ we can find a $z_{j} \in\left[y_{k+j}, x\right]$ with $f^{M(k, j)}\left(z_{j}\right)$ $=y$. As in Case 1 , since $\lim _{j \rightarrow \infty} y_{k+j}=x, x \in \alpha_{L}(y)$. By Lemma 2 and the fact that $x \in \alpha_{L}(x)$, we see that for every $y \in(x-\varepsilon, x], x \in s \alpha(y)$. This completes the proof of the claim.

To complete the proof of the lemma we must show that $x \in \Gamma$. Let $\delta>0$ be such that for every $y \in(x-\delta, x), x \in s \alpha(y)$. According to the proof of Claim 1, $x \in \omega_{L}(y)$ for some $y \in(x-\delta, x)$, and hence $x \in \omega_{L}(y) \cap s \alpha(y)$. Since $s \alpha(y) \subseteq \alpha(y), x \in \Gamma$.

The following lemma will complete the proof of the theorem.

Lemma 6. If $x \in \Gamma$ then $x \in s \alpha(x)$.

Proof. If $x \in R$ then, by Lemma 5, $x \in s \alpha(x)$. According to Xiong in [9],

$$
\Gamma=\left(\bigcup_{y \in I} \omega_{L}(y) \cap \alpha_{L}(y)\right) \cup\left(\bigcup_{y \in I} \omega_{R}(y) \cap \alpha_{R}(y)\right) \cup P .
$$

Thus we assume that $x \notin R$ and that $y$ has been chosen with $x \in \omega_{L}(y) \cap$ $\alpha_{L}(y)$. Let $\varepsilon>0$ be chosen so that if $n>0$ then $f^{n}(x) \notin(x-\varepsilon, x+\varepsilon)$. Let $\left(y_{i}\right)_{i=1}^{\infty}$ and $\left(z_{i}\right)_{i=1}^{\infty}$ be sequences with $y_{i} \in(x-\varepsilon, x), z_{i} \in(x-\varepsilon, x)$, $f^{n(i)}(y)=y_{i}, f^{m(i)}\left(z_{i}\right)=y, \lim _{i \rightarrow \infty} n(i)=\infty$, and $\lim _{i \rightarrow \infty} m(i)=\infty$. Let $L(i, j)=m(i)+n(j)$. Then $f^{L(i, j)}\left(z_{i}\right)=y_{j}$ for all $i>0$ and $j>0$.

Case 1. There exists $j>0$ such that $f^{L(i, j)}(x)>x+\varepsilon$ for an infinite number of $i$. Let $(h(i))_{i=1}^{\infty}$ be an increasing subsequence of positive integers such that $f^{L(h(i), j)}(x)>x+\varepsilon$. Since $f^{L(h(i), j)}\left(z_{h(i)}\right)=y_{j}$, it follows that

$$
(* * *) \quad\left[y_{j}, x+\varepsilon\right) \subseteq f^{L(h(i), j)}\left(\left[z_{h(i)}, x\right]\right) .
$$

To finish the proof by Lemmas 2 and Proposition 1, it suffices to show that for every $y \in\left(y_{j}, x\right), x \in \alpha_{L}(y)$.

Let $y \in\left(y_{j}, x\right)$. For every $i>0$, there exists $w_{i} \in\left(z_{h(i)}, x\right)$ with $f^{L(h(i), j)}\left(w_{i}\right)=y$. It follows that $x \in \alpha_{L}(y)$.

Case 2. If $j>0$ then there exists an integer $M(j)$ such that for $i>M(j)$, $f^{L(i, j)}(x)<x-\varepsilon$. 
To finish the proof in this case, we will show that if $y \in(x-\varepsilon, x)$ then, $x \in \alpha_{L}(y)$. Let $y \in(x-\varepsilon, x)$. Since $\lim _{i \rightarrow \infty} y_{i}=x$, we can find a $j>0$ with $x-\varepsilon>y<y_{j}$. For every $i>M(j)$ we have

$$
\left(x-\varepsilon, y_{j}\right] \subseteq f^{L(i, j)}\left(\left[z_{i}, x\right)\right) .
$$

Hence for every $i>M(j)$, we can find $w_{i} \in\left(z_{i}, x\right)$ with $f^{L(i, j)}\left(w_{i}\right)=y$. It follows that $x \in \alpha_{L}(y)$.

\section{ACKNOWLEDGMENTS}

The author would like to thank Professor Richard J. O'Malley and the referee for their many useful comments.

\section{REFERENCES}

1. J. Auslander and J. York, Interval maps, factors of maps, and chaos, Tohoku Math. J. 32 (1980), 177-188.

2. L. Block and E. Coven, w-limit sets for maps of the interval, Ergodic Theory Dynamical Systems 6 (1986), 335-344.

3. H. Chu and J.-C. Xiong, A counterexample in dynamical systems of the interval, Proc. Amer. Math. Soc. 97 (1986), 361-366.

4. W. A. Coppel, Continuous maps of the interval, xeroxed notes, IMA, Minnesota, 1984.

5. E. Coven and G. A. Hedlund, Continuous maps of the interval whose periodic points form a closed set, Proc. Amer. Math. Soc. 79 (1980), 127-133.

6. E. Coven and Z. Nitecki, Non-wandering sets of the powers of maps of the interval, Ergodic Theory Dynamical Systems 1 (1981), 9-31.

7. Z. Nitecki, Topological dynamics on the interval, Ergodic Theory Dynamical Systems II, College Park, MD 1979/1980, Progr. Math., vol. 21, Birkäuser, Boston, MA, 1982, pp. $1-73$.

8. A. Sharkovskiī, On a theorem of G. D. Birkhoff, Dopovīdī Akad. Nauk. Ukraīn. RSR Ser. A (1967), 429-432.

9. J.-C. Xiong, The attracting centre of a continuous self-map of the interval, Ergodic Theory Dynamical Systems 8 (1988), 205-213.

Department of Mathematics, Bradley University, Peoria, Illinois 61625

E-mail address: herol@bradley.bradley.edu 